\title{
Die Samen in der UdSSR
}

\author{
Historischer Überblick und Gegenwart
}

By Tatjana Lukjantschenko

Die ersten schriftlichen Angaben über die Kola-Samen gibt es im Bericht von Otar, der sich auf das IX. Jahrhundert bezieht. In den russischen Quellen erscheint die Benennung ,LLo" " seit dem Ende des XIV. Jahrhunderts. Seit dem XV. Jahrhundert beginnen die Angaben über die Lappen in verschiedenen russischen Urkunden zu erscheinen, wie z. B. in den Nowgoroder Kanzleibüchern die Erwähnungen von „Dikoi“", „Leschei“, „Lop“ und ,Lopljany“ etc.

In dieser Periode, d. h. seit dem Ende des I. Jahrtausends und der ersten Hälfte des II. Jahrtausends nahmen die Kola-Lappen ein bedeutend grösseres Territorium als heute ein. Sie besiedelten weite Gebiete des heutigen Kareliens, wovon die Toponymik sowie die in verschiedenen Quellen vorhandenen Erwähnungen der ,Lopskie pogosty“ in Saoneshje, d.h. dem Lande nördlich vom Onegasee, zeugen. In südöstlicher Richtung erstreckte sich das Territorium der Lappen bis zu den Ländereien des Klosters Murom. Die östliche Grenze der Niederlassung der Lappen verlief wahrscheinlich zu jener Zeit westlich vom Fluss Mesen.

Nach ihrem Wirtschafts- und Kulturtyp waren die Lappen in der ersten Hälfte des II. Jahrtausends hauptsächlich Renjäger, Elchjäger, Pelztierjäger; auch waren sie Fischer an den Binnengewässern und an der Meeresküste. Sie trieben auch wahrscheinlich ein wenig Renzucht. Man verwendete Rene als ,Mantschiki“" bei der Renjagd, um die Tiere anzulocken. Man kann sich denken, dass schon in dieser Zeit die Zucht von TransportRenen sich zu entwickeln beginnt, zuerst für Lasten und später für $\mathrm{Ge}-$ spanne.

Wie es verschiedenes Material bezeugt, konnte man bei den Lappen zu jener Zeit eine Teilung in zwei Gruppen entsprechend den Gebieten ihrer Niederlassung und ihren wirtschaftlichen und kulturellen Besonderheiten beobachten. Eine Gruppe stellten die Küsten-Lappen oder See-Lappen dar, die in der Nähe der Murmansk-Küste der Barentssee wohnten. Sie unternahmen nur kürzere Wanderungen und hatten eine Neigung zum Meeresfischfang. Die andere Gruppe stellten die Wald-Lappen dar, die die südlichen Wald- und Wald-Tundragebiete besiedelten. Die Lebensweise dieser 
Lappen war viel unsteter. Ihre Hauptbeschäftigungen waren die Jagd und der Fischfang in den Binnengewässern. Wahrscheinlich begann sich gerade bei dieser Gruppe die Renzucht zuerst zu entwickeln. Man kann feststellen, dass eine ähnliche Teilung in Küstengruppen und Waldgruppen auch anderen Völkern der arktischen Zone Eurasiens eigen war, wie z. B. den Nenzen und Enzen.

Um die Wende vom I. zum II. Jahrtausend stellten die Lappen, die die Halbinsel Kola und Karelien besiedelten, wahrscheinlich recht wenige verwandtschaftliche Kollektive dar, die ein unstetes Leben führten und mit anderen Völkern wenig Kontakt hatten.

In den Quellen werden drei territoriale Gruppen von Lappen erwähnt, und zwar die ,Terskaja Lop" der östlichen Bezirke der Halbinsel Kola, die , ,Kontschanskaja Lop" der westlichen Bezirke der Halbinsel und die , Leschaja Lop" von Karelien.

Aber schon in diesem Stadium kann man von den Kontakten der Lappen mit anderen Völkern sprechen, die zuerst hauptsächlich den Charakter von Tauschhandel trugen. So kann man seit dem XII. Jahrhundert von den Kontakten der Lappen mit Russen sprechen, die in den Norden eindringende Nowgoroder waren. Ungefähr in dieselbe Zeit fallen die ersten Kontakte der Lappen mit Kareliern. Viele Jahre lang bis zum Jahre 1326 fuhren die Norweger zu den Lappen, die die Küste der Barentssee besiedelten, um Steuern einzutreiben ${ }^{1}$. Am Angang des II. Jahrtausends fanden wahrscheinlich auch Kontakte von Lappen im Osten ihres Areals, d. h. irgendwo im Mesen-Becken, mit den westlichen Gruppen der Samojeden statt, die damals aus Sibirien in den europäischen Norden eindrangen. Die Folge aller dieser Kontakte war, dass die Lappen die Schusswaffe, Kleidung aus Tuch sowie einige andere Besonderheiten in Wirtschaft und Kultur einführten.

Im Laufe des XV.-XVI. Jahrhunderts vollzog sich eine verstärkte Kolonisierung des Territoriums der Halbinsel Kola mit Russen. An der Terskund Murmanskküste entstanden die ersten russischen ständigen Siedlungen. Wahrscheinlich begann schon in dieser Zeit die Vermischung der Lappen mit Russen. Ein solcher Prozess vollzog sich zum Beispiel im Bezirk Ponoj, dort wo im Frühling der Fang von Fischen und anderen Meerestieren betrieben wurde, an dem ausser den Russen, die für diese Zeit aus verschiedenen Orten kamen wie ,Dwinjane“, ,,Kolmogorzi“", ,Saoneshane“, auch Lappen teilnahmen. Gegen Ende des XIX. Jahrhunderts waren die Lappen von Ponojskij Pogost von den Russen völlig assimiliert.

${ }^{1}$ Im Jahre 1326 unterschreiben Norwegen und Nowgorod einen Friedensvertrag, in dem die Norweger die nördliche Küste der Kola-Halbinsel als russisches Territorium anerkannten. 
Aber die übrigen Gruppen der Kola-Lappen schlossen bis zum XX. Jahrhundert ziemlich selten Ehen mit den Angehörigen anderer Völker.

Nach dem Material des XIX. Jahrhunderts kann man Niederlassungen von Kola-Lappen schon nur noch auf der Kola-Halbinsel beobachten. Aus dieser Zeit gibt es viele Beschreibungen des Lebens und der Lebensweise der Kola-Lappen. In dieser Zeit erscheinen auch ernsthafte wissenschaftliche Werke, von denen vor allem die Monographie des bekannten russischen Ethnographen Nikolaj Charusin Russkije lopari zu erwähnen ist, die im Jahre 1890 erschien. Das Buch von N. Charusin, das eine ausführliche Beschreibung der Wirtschaft, der materiellen Kultur, der Bräuche und des Glaubens der Kola-Lappen damaliger Zeit enthält, hat bis in unsere Zeit nicht an Bedeutung verloren.

Am Anfang des XX. Jahrhunderts bildeten die Kola-Samen drei Wirtschafts- und Kulturgruppen:

1) die Küstensamen wohnten an der Küste der Barentssee und an der Weissmeerküste. Ihre Hauptbeschäftigungen waren Dorschfang und Lachsfang in den Flussmündungen und Netzfang von Meerestieren an der Küste. Die Renzucht spielte bei ihnen eine Nebenrolle.

2) die Samen, die in östlichen Innenbezirken der Halbinsel wohnten. Ihre Hauptbeschäftigung war zu damaliger Zeit die Renzucht. Eine untergeordnete Rolle spielten der Fischfang an den Seen und Flüssen sowie die Pelztierjagd (Fuchs, Marder, Eisfuchs u.s.w.)

3) die Samen, die in den westlichen Innenbezirken der Halbinsel Kola wohten, bildeten eine besondere Gruppe. Ihre Wirtschaft gründete sich gleichermassen auf Fischfang und Renzucht. Eine Nebenrolle spielte die Pelztierjagd.

Die ganze samische Bevölkerung der Halbinsel Kola bestand, nach den Angaben von N. Charusin, am Ende des XIX. Jh. aus 17 samischen Gruppierungen (Lowosero, Jokanga, Semiostrowje, u.s.w.), die wahrscheinlich territoriale Vereinigungen vom Typ der Nachbargemeinde darstellten.

Die Gesellschaften hatten ständige Siedlungen - pogosti (Winter- und Sommersiedlungen) und ausserdem noch einige Frühlings- und Herbstplätze, zu denen verwandte Familien zum Fischfang fuhren.

Die Gesellschaften besassen Jagd- und Weideplätze, und die Familien besassen Fischfangplätze, wobei ihr Besitzrecht für bestimmte Plätze periodisch überprüft wurde.

Am Ende der 80er Jahre des XIX. Jahrhunderts erschienen auf der Halbinsel Kola die Komi-Ishemzen die mit ihren Renherden vom Petschora-Fluss kamen. Die Komi bekamen von der samischen Gesellschaft Lowosero die Erlaubnis, sich in ihrer Winter-Pogost niederzulassen. 
Später entstanden Komi-Siedlungen an den Flüssen Ponoj, Iwanowka und Krasnoshelje.

Die Kontakte der Samen mit den Komi, die nach der Kollektivisierung besonders intensiv wurden, spielten im Leben der Samen, und zwar in der Entwicklung ihrer Renzucht, des Rentransports und der Tuchkleidung eine grosse Rolle.

Das XX. Jahrhundert hat grosse Veränderungen in das ökonomische, soziale und kulturelle Leben der Samen gebracht. Das ist vor allem auf die Arbeit zurückzuführen, die der Sowjetstaat seit den ersten Tagen der Errichtung seiner Macht auf Kola durch Hilfe für die angestammte Bevölkerung und Beseitigung ihrer ökonomischen und kulturellen Rückständigkeit geleistet hat.

Eine grosse Rolle im Leben der Samen und für die Verstärkung ihrer Kontakte mit anderen Völkern spielt die Kollektivisierung und die Vereinigung der Einzelwirtschaften zu Kolchosen, die gewöhnlich hinsichtlich ihrer völkischen Zusammensetzung gemischt waren (es vereinigten sich Wirtschaften der Samen, Komi, Karelier, Russen, u. a.).

In den 20er Jahren begann auf der Halbinsel Kola eine intensive Entwicklung der Industrie - Bergbau, Elektrizitätserzeugung, Fischereiwirtschaft u. a. - was einen grossen Zustrom von Menschen aus verschiedenen Gebieten des Landes hervorrief. Dieser Prozess vollzog sich dann besonders intensiv in den 50er bis 60er Jahren und er dauert immer noch an. Das Resultat: Das abgeschlossene und isolierte Leben der Samen nahm ein Ende. Die Verbindungen der Samen mit Nachbarenvölkern wurden stärker, und es begann ein Prozess der Annäherung der Kulturen.

Die gegenwärtige Zahl der Kola-Samen beträgt nach der Volkszählung 19791900 Menschen. Sie leben vorwiegend im Bezirk Lowosero bei Murmansk. Das Hauptzentrum ihrer Niederlassung ist die Siedlung Lowosero. Ihre Hauptbeschäftigung ist die Renzucht, der wichtigste Wirtschaftszweig der Sowchose ,Tundra“. Ausser den Samen sind in der Renzucht auch Komi und Nenzen beschäftigt.

Die Kola-Samen leben unter den Bedingungen enger zwischenethnischer Kontakte. Trotzdem verliert sich diese kleine Völkerschaft nicht in den grösseren benachbarten Völkern, sondern entwickelt ihre Kultur weiter.

So wurden zum Beispiel in der Renzucht, die heute ein staatlicher Wirtschaftszweig ist, einige traditionelle Verfahren der Samen beibehalten oder weiterentwickelt (der Gebrauch von Zäunen, die Bevorzugung, Rentiere zu Fuss und nicht von Narten aus zu weiden u.a.m.).

Trotz einer starken Verbreitung der russischen Sprache als Sprache des zwischennationalen Verkehrs in allen Sphären des Lebens - im Familienle- 
ben, in der Produktion, im Gesellschaftsleben - bemühen sich die Samen, ihre Muttersprache zu erhalten.

Samisch wird heute hauptsächlich als Sprache im häuslichen Leben gebraucht. Aber in den letzten Jahren verbreitete sich sein Anwendungsbereich etwas weiter. So hat zum Beispiel der lokale Rundfunk begonnen, einige Sendungen in samisch auszustrahlen. Es wurde eine samische Schriftsprache geschaffen. Man hat auch angefangen, Samisch in den Schulen zu unterrichten. Es wurden Sammelbände mit Gedichten der samischen Dichter O. Woronowa und A. Bazanow herausgegeben. Das Volkslied- und Tanzensemble in Lowosero singt samische Lieder in samischer und russischer Sprache.

Bei den Samen erhält sich das ethnische (nationale) Selbstbewusstsein, d. h. das Bewusstheit der Zugehörigkeit zu ihrer Nation oder, wie man sagen kann, die samische Identität.

Bei den Kola-Samen vollziehen sich auch andere Prozesse, die für die Völker der UdSSR charakteristisch sind. Es ist bekannt, dass für das ganze Land eine allmähliche Zunahme der Stadtbevölkerung im Gegensatz zur Landbevölkerung typisch ist. Bei den Samen, die Anfang unseres Jahrhunderts praktisch nur auf dem Lande wohnten, beträgt zur Zeit die Stadtbevölkerung über $20 \%$. Das sind hauptsächlich die Mitarbeiter im Schulen, medizinischen und anderen Anstalten, Abkömmlinge aus national-gemischten Ehen, die Städter geheiratet haben.

Wie bei anderen Völkern der UdSSR sind bei den Samen Migrationsprozesse zu beobachten, und zwar nicht nur in der Richtung aus dem Dorf in die Stadt, sondern auch über das Murmansker Gebiet und sogar über die Grenzen der RSFSR hinaus. Während Anfang des Jahrhunderts ausserhalb des Murmansker Gouvernements weniger als 0,3\% Samen wohnten, wohnen dort heute fast $20 \%$ der Samen.

Zum Abschluss kann man sagen, dass für das gegenwärtige Leben der Kola-Samen ebenso wie für andere Völker der UdSSR folgende Prozesse charakteristisch sind: auf der einen Seite die Verstärkung der Migration, die Zunahme der Stadtbevölkerung, eine Verbreitung der russischen Sprache, auf der anderen Seite die Erhaltung der Muttersprache und der nationalen Identität sowie eine Verstärkung des Interesses an ihrer traditionellen Kultur. Man kann behaupten, dass die Kola-Samen eine grosse ethnische Festigkeit aufweisen und sich als Ethnos erhalten. 